\title{
SNR in MI Catheter Receivers for MRI
}

\author{
E.M. Kardoulaki, R.R.A. Syms, Senior Member IEEE, I.R. Young, M. Rea
}

\begin{abstract}
Internal coils have a signal-to-noise ratio (SNR) advantage during magnetic resonance imaging (MRI). However, coils with continuous cables are generally unsafe, due to the risk of RF heating. Segmented cables such as magneto-inductive (MI) waveguides should introduce inherent safety at the price of increased noise, from both the cable and the body. Here we derive analytical SNR expressions for both types of noise, develop a model to compare the SNR of different types of receiver, and validate the model with data from imaging experiments at 3T. Experiments and theory confirm that body noise does not prevent a SNR gain compared to an 8-element external coil, even when a long section of waveguide is loaded with tissue.
\end{abstract}

\section{Index Terms - Noise, SNR, MRI, Magneto-inductive Waveguide}

\section{INTRODUCTION}

I NTERNAL magnetic resonance imaging (MRI) using small coils has been demonstrated [1-3], but clinical acceptance is relatively slow. There are difficulties associated with delivery into the patient, MR compatibility of endoscopic tools and the non-uniform sensitivity of the coils. Another important factor is patient safety due to the risk of tissue heating. Local heating can result from electrical excitation of standing waves on long conductors immersed in tissue [4], and inadequate decoupling from the $B_{1}$ magnetic field can cause further heating [5]. To avoid this problem, MR-safe cables incorporating chokes [6] or transformer segmentation in coaxial transmission lines $[7,8]$ have been proposed but the designs are generally complex and require additional discrete components for construction.

To improve clinical utility, we have developed MR receiver systems based on magneto-inductive (MI) waveguides [9], originally for use at $1.5 \mathrm{~T} \mathrm{[10]} \mathrm{and} \mathrm{later} \mathrm{at} \mathrm{3T} \mathrm{[11].} \mathrm{Heating}$ due to standing wave resonances is minimized by the absence of a continuous electrical path. In contrast to [7], the systems consist only of coupled L-C resonators and do not contain any transmission line sections. However, as in [8], figure-of-eight shaped elements are used to reject uniform $B_{1}$ fields.

The entire system is fabricated as a smooth, flexible PCB (printed circuit board) with integrated inductors and capacitors, using the substrate as a dielectric interlayer. Tuning is achieved by mechanical trimming of the capacitors. Matching is achieved entirely through control of mutual inductances and additional discrete components for tuning and matching are not required. The circuit is mounted on the

Manuscript received July 2015. This work was supported by the Welcome Trust ISSF Networks.

M. Rea is with the Department of Radiology, Imperial College Healthcare NHS Trust, Paddington, London W2 1NY, United Kingdom. All other authors are with the Electrical and Electronics Engineering Department, Imperial College London. London SW7 2AZ, U.K. (e-mail: evi.kardoulaki@ic.ac.uk, r.syms@ic.ac.uk, youngimarl@aol.com, marc.rea@ic.ac.uk) outside of a hollow catheter designed for future imaging of the biliary duc tal system. The catheters are flexible, with a demonstrated bending radius of $5 \mathrm{~mm}$ [10], and can pass over a guide wire and through the biopsy channel of a nonmagnetic duodenoscope [12]. A preliminary investigation of $\mathrm{B}_{1}$ and E-field coupling at 1.5T has also been carried out, and no evidence of significant coupling effects was found [10]. A theoretical model explaining the underlying reason has been presented [13], together with preliminary experimental results showing relative immunity to RF heating.

Here we investigate the trade-off between safety and SNR gain that could limit their practical use. Thin-film MI waveguides are lossy, low frequency metamaterials [14] and consequently suffer from thermal noise arising from conductor resistance $[15,16]$. In addition, because they can detect RF signals along their entire length [10], they are also susceptible to body noise [17]. The effect on SNR of proximity to a conductive sample has been considered for conventional coils [18-24] but not for MI-based receivers.

We develop a model for noise by approximating internal and external coils as rectangular elements inside and outside the body respectively. We then compare the SNR achievable with MI receivers, internal coils with a standard RF coaxial output and external coils. Theory is validated with experimental results at 3T. The operation of the catheter receiver is explained in Section II and a noise analysis is provided. Expressions for the detected signal and the body noise are also derived. Model validation is presented in Section III and a theoretical comparison of the SNR of the different coil types is carried out. The results are discussed in Section IV and conclusions are drawn in Section V.

\section{THEORY}

\section{A. Thermal noise in MRI detection systems}

Ignoring amplification and lossless matching networks, loss and noise in any MRI detection scheme can be represented as shown in Fig. 1. The intrinsic SNR is determined by the nuclear magnetic resonance (NMR) signal $V_{\mathrm{s}}$ and the body noise $V_{N B}$, derived from an equivalent resistor $R_{B}$. Due to their restricted field-of-view (FOV), internal coils have lower values of $R_{B}$ than external coils and consequently a higher intrinsic SNR. The detected SNR is modified by loss and noise in the coil and interconnect, both of which can be represented by noise sources $V_{N C}$ and $V_{N I}$ due to resistances $R_{C}$ and $R_{I}$ respectively. Internal coils have larger values of $R_{C}$ than external coils, but these are typically comparable to the small $R_{B}$ values, so the intrinsic SNR is not degraded significantly. Conventional cables do not further degrade the SNR by much since they have low values of $R_{I}$, but without modification are unsafe for internal use. MI cables have larger $R_{I}$, but provide potential safety advantages. In the following sections, we provide a quantitative proof. 


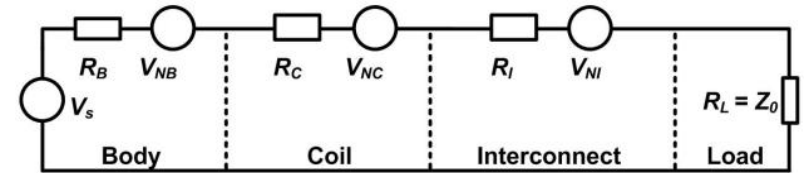

Fig. 1: MRI signal detection: sources of signal, loss and noise.

\section{B. Electrical equivalent models of different $R F$ receivers}

Fig. 2a shows a conventional receiver connected to a transmission line of impedance $Z_{0}$. It is based on a lossy inductor $L$ with resistance $R=R_{C}+R_{B}$ and is resonant at angular frequency $\omega_{0}=1 / \sqrt{ }(L C)$, where $1 / C=1 / C_{T}+1 / C_{M}$, and $C_{T}$ and $C_{M}$ are tuning and matching capacitors. The line is lossless and matched to the system input impedance. Matching of the receiver to the transmission line requires $1 /\left(\omega_{0} C_{M}\right)=$ $\sqrt{ }\left(R Z_{0}\right)$. The receiver can be internal or external, depending on its position with respect to the body.

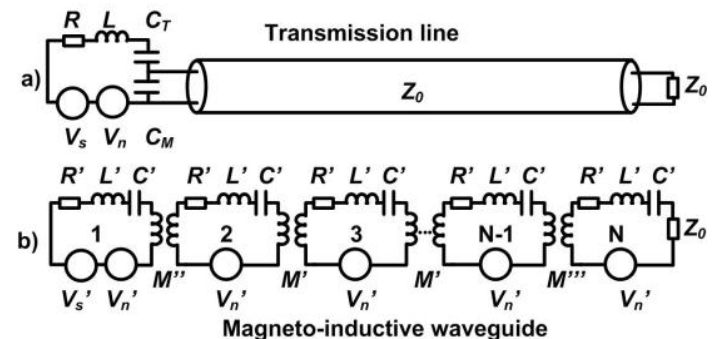

Fig. 2: Equivalent circuit models of different receivers: a) conventional internal or external receiver and b) MI catheter receiver.

Fig. $2 \mathrm{~b}$ shows an alternative magneto-inductive internal receiver consisting of a resonant detector, a MI cable and a coupling transducer. The complete system is an array of $N$ resonant elements with inductance, resistance and capacitance $L^{\prime}, R^{\prime}=R_{C}{ }^{\prime}+R_{B}{ }^{\prime}$, and $C^{\prime}$. In practice, $R_{B}{ }^{\prime}$ will not be the same for all elements either because the catheter is only partly loaded with tissue or due to tissue inhomogeneity. The first element of the cable is magnetically coupled to the detector by mutual inductance $M$ '. The last element is coupled to the transducer by mutual inductance $M$ ". . All other elements are coupled to their next neighbours with mutual inductance $M$ '.

To begin with, considering only nearest neighbour interactions in an infinite MI waveguide, with identical resonant elements $\left(L^{\prime}, R^{\prime}, C^{\prime}\right)$ and mutual inductances $\left(M^{\prime}\right)$, the currents $I_{n-1}, I_{n}$ and $I_{n+1}$, at angular frequency $\omega$ must satisfy the recurrence relation $\left(R^{\prime}+\mathrm{j} \omega L^{\prime}+1 / \mathrm{j} \omega C^{\prime}\right) I_{n}+\mathrm{j}$ $\omega M^{\prime}\left(I_{n-1}+I_{n+1}\right)=0$ [9]. Assumption of a wave solution $I_{n}=I_{0}$ $\exp (-\mathrm{j} n k a)$ for the current in the $\mathrm{n}^{\text {th }}$ element leads to: 1 $\left(\omega_{0} / \omega\right)^{2}-\mathrm{j} / Q+\kappa \cos (k a)=0$, with $I_{0}$ the wave amplitude, $k$ the propagation constant, $a$ the element length, $\omega$ the angular frequency, $\omega_{0}=1 / \sqrt{ }\left(L^{\prime} C^{\prime}\right)$, the angular resonant frequency, $Q$ $=Q_{0} \omega / \omega_{0}$ where $Q_{0}=\omega_{0} L^{\prime} / R^{\prime}$, the Q-factor and $\kappa=2 M^{\prime} / L^{\prime}$, the coupling coefficient. Assuming a complex propagation constant $k=k^{\prime}-\mathrm{j} k$ ' and a small value of $k$ ' $a$, we obtain:

$$
\begin{aligned}
& 1-\left(\omega_{0} / \omega\right)^{2}+\kappa \cos \left(k^{\prime} a\right)=0 \\
& k^{\prime \prime} a=1 /\left\{\kappa Q \sin \left(k^{\prime} a\right)\right\}
\end{aligned}
$$

The upper equation is the loss-less dispersion relation, and shows that for $\kappa>0$, propagation is permitted in a frequency band $1 / \sqrt{ }(1+\kappa) \leq \omega_{0} / \omega \leq 1 / \sqrt{ }(1-\kappa)$. The lower equation is the approximate loss variation. The finite $Q$ factor introduces attenuation and allows propagation out-of-band. Loss is minimized at resonance, when $k^{\prime} a=\pi / 2$, and is inversely proportional to both $\kappa$ and $Q_{0}$.

The characteristic impedance of the cable is $Z_{M}=\mathrm{j} \omega M^{\prime}$ ' $\sin (\mathrm{ka})$ which for low loss reduces to the real value $Z_{O M}=$ $\omega_{0} M^{\prime}$ at resonance. The detector should be matched to $Z_{O M}$ while the last cable element should be matched to the scanner input. These conditions require that $\omega_{0} M^{\prime \prime}=\sqrt{ }\left(R^{\prime} Z_{O M}\right)$ and $\omega_{0} M^{\prime \prime},=\sqrt{ }\left(Z_{0} Z_{O M}\right)$. Although $Z_{O M}$ can be made equal to $50 \Omega$, it will usually differ from this value. Matching to the scanner is then achieved by adjusting the number of turns or position of a demountable transducer [10].

When the tip is used for detection, the induced signal is transmitted along the guide as a MI wave, until the transducer couples it to the scanner electronics. Magnetization from tissue elsewhere can induce signals in any element of the cable. In this case, the induced signal will excite waves that propagate in quadrature towards the transducer, enabling imaging along the entire length of the catheter.

In more arbitrary cases, where the elements may each be different, $N$ coupled circuit equations relating the currents and voltage sources can be derived using Kirchhoff's voltage law. These can be written in the form $\boldsymbol{V}=\boldsymbol{Z} \boldsymbol{I}$, where $\boldsymbol{V}$ and $\boldsymbol{I}$ are $\mathrm{N}$ element column vectors containing the voltages and currents and $\boldsymbol{Z}$ is an $N$ x $N$ matrix of impedances. The unknown currents may be found by matrix inversion, as $\boldsymbol{I}=\boldsymbol{Z}^{-1} \boldsymbol{V}$, and the transmission and reflection coefficients may then be extracted and converted into scattering parameters.

\section{Noise analysis}

Methods based on attenuation are conventionally used to calculate noise in impedance-matched systems containing only a single noise source. Here we use a method viable for more complex networks such as MI waveguides containing multiple sources $[15,16]$. For non-uniform systems (for example, with different numbers of elements immersed in tissue), the equations must generally be solved numerically; however, analytic approximations are possible for uniform systems.

Noise in the conventional receiver (Fig. 2a) can be modeled in terms of random voltages due to thermal noise from the coil and body. At absolute temperature $T$, this noise has a RMS value in a bandwidth $B$ of $V_{n}=\sqrt{ }\left(4 k_{B} T B R\right)$, where $R=R_{C}+R_{B}$, $R_{C}$ and $R_{B}$ are the effective resistances of the coil conductors and the body, and $k_{B}$ is Boltzmann's constant. If a RMS signal voltage $V_{s}$ is induced in the coil, the amplitude SNR is then given by $S=\left|V_{s} / V_{n}\right|$. This value is unaffected by the output cable, if it can be considered lossless on account of its size; however smaller cables may have a significant effect.

Noise in the MI receiver (Fig. 2b) can be modeled in a similar way in terms of random voltages in each resonant element. Again the RMS value of $V_{n}{ }^{\prime}$ is $\sqrt{ }\left(4 k_{B} T B R R^{\prime}\right)$, where $R$ ' $=R_{C}{ }^{\prime}+R_{B}{ }^{\prime}$. For simplicity, we assume complete immersion in a homogeneous medium, so that the same $R_{B}$ ' loads all elements. We also ignore the possibility of signal voltages $V_{s}$ ', in loops other than the first.

At resonance, the matched system with $Z_{O M}=Z_{0}$ can be 
represented as a resistance $R^{\prime}$ in the first loop. The current in this loop due to the signal source $V_{s}^{\prime}$ must therefore be $I_{l s}=$ $V_{s}^{\prime} / 2 R^{\prime}$. Now the circuit equation of loop 1 at resonance is: $R^{\prime} I_{1 s}+\mathrm{j} \sqrt{ }\left(R^{\prime} Z_{0}\right) I_{2 s}=2 R^{\prime} I_{I s}$. As a result, the current in the second loop must be $I_{2 s}=-\mathrm{j} V_{s}^{\prime} /\left[2 \sqrt{ }\left(R^{\prime} Z_{0}\right)\right]$. From (1), the attenuation term can be written as $k^{\prime \prime} a=1 / \kappa Q_{0}=R^{\prime} / 2 Z_{0}$. Consequently, the current in the $N^{\text {th }}$ resonator is:

$\left|I_{N s}\right|=V_{s}^{\prime} /\left(2 \sqrt{R^{\prime} Z_{0}}\right) \exp \left[-(N-2) R^{\prime} / 2 Z_{0}\right]$

The noise current at the load due to the $V_{n}$ ' in element 1 is:

$\left|I_{N n}\right|=V_{n}^{\prime} /\left(2 \sqrt{R^{\prime} Z_{0}}\right) \exp \left[-(N-2) R^{\prime} / 2 Z_{0}\right]$

The amplitude SNR is $S^{\prime}=\left|I_{N S}\right| I_{N n} \mid$, an expression analogous to the result for the conventional receiver. In reality, each noise source will generate a noise wave that travels to the load and these will add to the noise above, decreasing the SNR. To illustrate this we consider the noise source in element 2 . Since the sections of the cable on either side are matched, they may be represented in the loop simply as impedances $Z_{0}$. Ignoring self-resistance, the noise current $I_{2 n}$ due to this source in loop 2 at resonance is $I_{2 n} \approx V_{n}{ }^{\prime} / 2 Z_{0}$. The wave travelling towards the detector can be ignored, while the noise current at the load is:

$\left|I_{N n}\right|=\left(V_{n}^{\prime} / 2 Z_{0}\right) \exp \left[-(N-2) R^{\prime} / 2 Z_{0}\right]$

Similar terms will be generated by noise in elements $3 \ldots N$. The total noise can be found by incoherent summation of the term in (3) and a set of terms from (4), as:

$\left|I_{N n}\right|^{2}=\left(V_{n}{ }^{2} / 4 R^{\prime} Z_{0}\right) \exp \left[-(N-2) R^{\prime} / Z_{0}\right] F$

where $F$ is the noise factor, given by:

$$
F=1+\left(R^{\prime} / Z_{0}\right) \exp \left[(N-2) \frac{R^{\prime}}{Z_{0}}\right] \frac{1-\exp \left[-(N-1) R^{\prime} / Z_{0}\right]}{1-\exp \left(-R^{\prime} / Z_{0}\right)}
$$

The SNR at the load is then $S^{\prime}=\left|V_{s}^{\prime} / V_{n}^{\prime}\right| \times 1 / \sqrt{ } F$. This is consistent with standard theory [15], but must be compared with the numerical solution. To do so, the total noise power at the load is determined by summing the powers due to each noise source in Fig. 2b. We have found that a MI coil resonant at $127.6 \mathrm{MHz}$ with $N=20, Z_{0 M}=50 \Omega, \kappa=0.6, Q_{0}=70$, and $a$ $=10 \mathrm{~cm}$, has $F=1.45$ when $R^{\prime}=1 \Omega$. This value deviates by only $0.3 \%$ from the numerical result, largely due to the approximation previously mentioned.

\section{Body loading in a $2 D$ system}

We now derive an analytical expression for the equivalent resistance per unit length seen by a long, thin, rectangular coil due to body loading. We adopt the standard approach of reciprocity and ignore dielectric losses. However, because of the complexity of the geometry, we make significant approximations. Firstly, we ignore variations along the length of an immersed system. These can occur at the ends of a coil, due to field spreading, and also over a larger distance scale due to standing waves in the interconnect [24]. The aspect ratio of the MI receiver and the fact that we use an impedancematched detector, designed to prevent reflections, justify this approximation. In addition, locally uniform axial sensitivity has been observed experimentally.

Secondly, we make extensive use of quasi-static approximations that are likely to lose validity at high frequencies. Strictly the conductors support surface waves of the type originally investigated by Goubau [25] who solved the full electromagnetic problem of a cylindrical wire immersed in a lossy dielectric medium, with and without an insulating coating. He found that the coating had a significant effect on the electric field, considerably reducing the influence of the surrounding medium. As a result, the electric field rises from near zero in the conductor to a high value at the outer edge of the insulating coating, and then decays into the surrounding medium.

This rigorous approach has been used in recent models of RF-induced heating of lead wires during MRI [26]. Unfortunately, the geometry of our receiver makes a rigorous electromagnetic calculation extremely difficult. The circuit does not use cylindrical conductors but thin, rectangular ones. In addition, the insulating layer is not conformal to the conductor cross-section; instead, the two conductors are mounted on either side of a cylindrical scaffold, and surrounded by a thin cylindrical sheath. Thus, there is no simple geometry (such as cylindrical co-ordinates) to aid a full field calculation. In the derivation that follows, we therefore use simplifications that, although approximate, still allow useful analytical expressions to be derived.

We assume the geometry of Fig. 3a, which shows a system comprising a coil immersed in a homogeneous body torso and an external coil, consisting of anterior and posterior parts, resting on the outside of the torso. Fig. $3 \mathrm{~b}$ is a cross-section of the system indicating the fully immersed internal coil. A corresponding view of the same system featuring the external coil is shown in Fig. 3c.

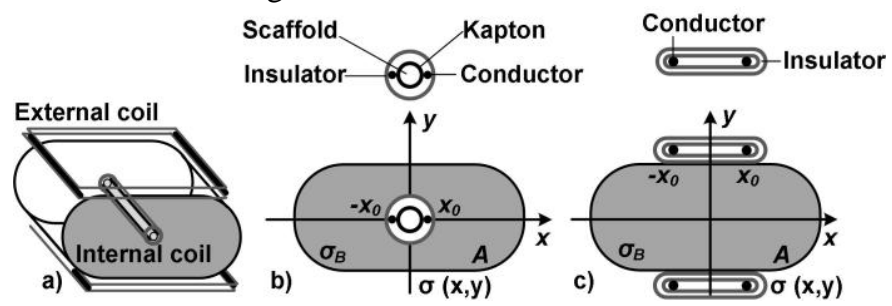

Fig. 3: a) System for the calculation of $R_{B}$ showing an external coil, and an internal coil; Cross-section for $b$ ) the internal coil and c) the external coil.

In each case, the coils are modelled as pairs of parallel cylindrical wires oriented in the $z$-direction, passing through the points $\left( \pm x_{0}, 0\right)$ and carrying currents $\pm I$. However, generally, there may be an array of such coils. Although, MI receivers are planar thin-film circuits, with inductors formed by rectangular tracks, at large distances the conductor shape can be ignored and a cylindrical wire approximation used. This is also generally valid for the external coils.

The origin of the co-ordinate system is chosen depending on 
the coil type and body geometry. The conductors generate a magnetic field which fills all space defined by the Cartesian co-ordinate system with permeability that of free space $(\mu=$ $\left.\mu_{o}\right)$ and a spatially varying conductivity $\sigma=\sigma(x, y)$. This is equal to $\sigma_{B}$ inside the conductive body of area $A$ and zero everywhere else. By choosing the variation of $\sigma$ appropriately, the effects of the catheter scaffold, the insulating sheath and other experimental features of later MRI arrangements (which involve multiple phantoms), can easily be considered. For uniform media, this geometry can conveniently be described entirely in terms of the specification of the exact area $A$.

We use the quasi-static approximation to determine the magnetic field, the curl equation to calculate the electric field and Poynting's theorem to estimate the power dissipated in the body. Although the first two assumptions must result in inaccurate expressions for the electric field, especially in the insulator, we do not anticipate that these will affect the dissipated power strongly, since we explicitly exclude in this calculation the insulator and the region enclosed by it.

The current in the conductors, the corresponding magnetic field and the accompanying electric field can be expressed as $\boldsymbol{I}(t)=\operatorname{Re}\{I \exp (\mathrm{j} \omega t)\}, \underline{\boldsymbol{H}}(t)=\operatorname{Re}\{\underline{H} \exp (\mathrm{j} \omega t)\}$ and $\underline{\boldsymbol{E}}(t)=\operatorname{Re}\{\underline{E}$ $\exp (\mathrm{j} \omega t)\}$ respectively, so that $\underline{\boldsymbol{H}}$ and $\underline{\boldsymbol{E}}$ represent time varying fields and $\underline{H}$ and $\underline{E}$ the corresponding time independent fields. For a single wire at the origin, the magnetic field is $\underline{H}=$ $(I / 2 \pi r) \underline{\theta}$ where $r=\sqrt{ }\left(x^{2}+y^{2}\right)$ and $\underline{\theta}$ is a unit vector in the tangential direction. For two wires located at $\left( \pm x_{0}, 0\right)$, the $x$ and $y$-components of the magnetic field are:

$$
\begin{aligned}
& H_{x}=-\frac{I}{2 \pi}\left(\frac{y}{\left(x+x_{0}\right)^{2}+y^{2}}-\frac{y}{\left(x-x_{0}\right)^{2}+y^{2}}\right) \\
& H_{y}=\frac{I}{2 \pi}\left(\frac{x+x_{0}}{\left(x+x_{0}\right)^{2}+y^{2}}-\frac{x-x_{O}}{\left(x-x_{0}\right)^{2}+y^{2}}\right)
\end{aligned}
$$

From Ampere's law, the electric field must lie in the $z$ direction. Employing Faraday's law and using (7), we obtain $E_{z}=\left(\mathrm{j} \omega \mu_{0} I / 4 \pi\right) \log _{\mathrm{e}}\left\{\left(\left(x+x_{0}\right)^{2}+y^{2}\right) /\left(\left(x-x_{0}\right)^{2}+y^{2}\right)\right\}$. From Poynting's theorem, the power per unit depth dissipated in the torso in Fig. 3 is $P_{B}=\left(\sigma_{B} \omega^{2} \mu_{0}^{2} I^{2} / 32 \pi^{2}\right) \iint_{\mathrm{A}} \log _{\mathrm{e}}^{2}\left\{\left(\left(x+x_{0}\right)^{2}+\right.\right.$ $\left.\left.y^{2}\right) /\left(\left(x-x_{0}\right)^{2}+y^{2}\right)\right\} \mathrm{d} x \mathrm{~d} y$. Comparing this expression with the power in an equivalent per-unit length resistance yields $R_{B}$ as:

$$
R_{B}=\frac{\sigma_{B} \omega^{2} \mu_{0}^{2} x_{0}^{2}}{16 \pi^{2}} \iint_{A_{S}} \log ^{2} e\left(\frac{(u+1)^{2}+v^{2}}{(u-1)^{2}+v^{2}}\right) d u d v
$$

Here $u=x / x_{0}$, and $v=y / x_{0}$ are dimensionless co-ordinates and $A_{S}=A / x_{0}^{2}$ is the dimensionless torso area. In general, the integration above must be carried out numerically, over the area containing the conductive material. However, (8) suggests that provided the conductor dimensions scale with $x_{0}$ small coils see reduced body noise.

\section{E. Calculation of the detected signal}

We now derive an expression for the received signal. By reciprocity, the voltage induced in a coil by an elemental volume $v$ containing a collection of nuclear magnetic dipoles rotating about the $z$-axis at angular frequency $\omega_{0}$ is $V_{s}=$ $\mathrm{j} \omega_{0} \underline{\boldsymbol{B}}_{x y} . \underline{M}_{x y}$. Here $\underline{M}_{x y}$ is the transverse magnetization obtained by an applied $90^{\circ}$ pulse with the sample at thermal equilibrium, and $\underline{B}_{x y}$ is the flux density generated by the coil in the $x-y$ plane when excited by a unit dc current. Here $B_{0}$ is considered to be in the $z$-direction. In complex notation, the time-independent transverse magnetization can be described as $\underline{M}_{x y}=M_{0}\left(\underline{u}_{x}+\mathrm{j} \underline{u}_{y}\right)$, with $\underline{u}_{x}$ and $\underline{u}_{y}$ unit vectors in the $x$ - and $y$-directions and $M_{0}$ the equilibrium nuclear magnetization density of water protons at 3T, namely $9.33 \times 10-9(\mathrm{~J} / \mathrm{ml})$. The RMS value of the signal from $v \mathrm{ml}$ of a voxel is therefore:

$V_{s}(x, y)=\frac{\omega_{0} v \mu_{0} M_{0}}{\sqrt{ } 2}\left(\sqrt{H_{x}^{2}+H_{y}^{2}}\right)$

\section{F. Derivation of the $S N R$}

The RMS value of the noise voltage due to $R_{B}$ and $R_{C}$ can be expressed as $V_{n}=\sqrt{ }\left[4 k_{B} T \Delta f\left(R_{B}+R_{C}\right)\right]$, with $\Delta f$, the voxel bandwidth. Combining this result with (9), the SNR variations in the $x$ - and $y$-directions from the centre of an internal or an external detector become:

$\operatorname{SNR}(x, y)=\frac{\omega_{0} \mu_{0} v M_{0}}{\sqrt{2}} \frac{\left(\sqrt{H_{x}^{2}+H_{y}^{2}}\right)}{\sqrt{\left[4 k_{B} T \Delta f\left(R_{B}+R_{C}\right)\right]}}$

Since $M_{0}=N \gamma h^{2} \mathrm{I}\{\mathrm{I}+1\} \omega_{0} /\left\{12 \pi^{2} k_{B} T\right\}$, where $N$ is the number of resonant spins per unit volume, $\gamma$ is the gyromagnetic ratio, I the spin quantum number and $h$ Planck's constant, the SNR is proportional to $\omega_{0} /{ } \sigma_{B}$ for large external coils $\left(R_{B} \gg R_{C}\right)$ and to $\omega_{0}^{2}$ for small internal coils $\left(R_{C} \gg R_{B}\right)$, implying a significant improvement at a higher static magnetic field. At large distance $\left(\sqrt{ }\left(x^{2}+y^{2}\right)>x_{0}\right)$, the coil sensitivity reduces as $1 / r^{2}$. The SNR from a pixel in a MRI image is $\mathrm{SNR}_{\text {pixel }}=\mathrm{SNR} \sqrt{\mathrm{NEX}} / F_{s}$, with $F_{s}$, the system noise factor, assumed equal to 1 and NEX, the number of excitations [18].

\section{Magnetic Resonance IMAGing}

Here we compare the performance of a MI receiver with that of the best locally available array coil (8-element HD cardiac) and assess the validity of the theoretical SNR model. We present results from a phantom study in a $3 \mathrm{~T}$ GE Discovery MR 750 clinical scanner.

\section{A. SNR comparison between magneto-inductive internal receivers and array coils}

The MI receiver consists of 20 magnetically coupled figureof-eight shaped resonators formed by double-sided patterning of copper-clad Kapton. Fig. 4a shows a section of the thin-film circuit. The copper and Kapton thickness are $17.5 \mu \mathrm{m}$ and 25 $\mu \mathrm{m}$ respectively, the copper track width is equal to $0.5 \mathrm{~mm}$, and the inductor width is $3.3 \mathrm{~mm}$. Each element has length $a=$ $100 \mathrm{~mm}$ and a period of $50 \mathrm{~mm}$. The overlap of the first cable element with the detector is $10 \mathrm{~mm}$ to achieve matching. The overall catheter length is $1 \mathrm{~m}$. Fig. $4 \mathrm{~b}$ shows a section of the assembled MI cable, mounted on a catheter and insulated by a thin-film heat shrink sleeve resulting in a final OD of $3.2 \mathrm{~mm}$. 
Referring to Fig. $2 \mathrm{~b}$, the $20^{\text {th }}$ element is the demountable transducer, essentially a two-turn spiral inductor, secured in a split Perspex clamp. Fig. 4c shows the transducer, together with the proximal end of the catheter-mounted MI cable. The MI receiver is tuned and matched for operation at $127.6 \mathrm{MHz}$. Since it relies on its figure-of-eight elements for $\mathrm{B}_{1}$ decoupling, a non-magnetic PIN diode (MA4P7464F-1072, MACOM) was connected across the transducer to enable identification during the pre-scan test.

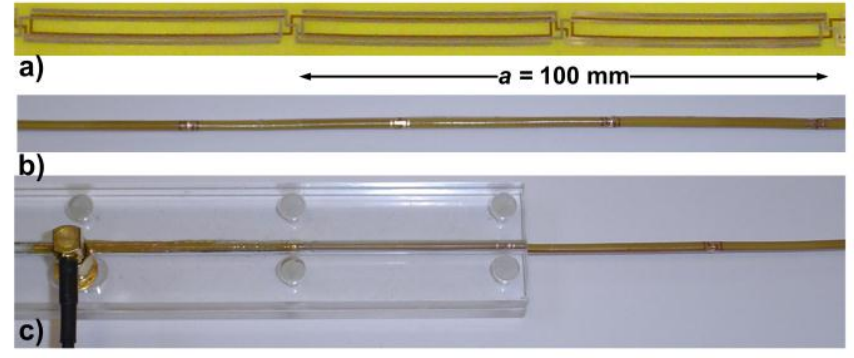

Fig. 4: Section of the MI catheter receiver: a) flat, b) assembled on catheter, c) demountable transducer and proximal end of the MI catheter receiver.

Fig. 5a shows the arrangement for imaging with the MI catheter receiver, which is partly inserted over $10 \mathrm{~cm}$ between two cuboid phantoms $(15 \mathrm{~cm}(\mathrm{~W}) \times 15 \mathrm{~cm}(\mathrm{H}) \times 35 \mathrm{~cm}(\mathrm{~L}))$ containing a tissue simulating solution $\left(3.368 \mathrm{~g} / \mathrm{L} \mathrm{NiCl}_{2} \cdot \mathrm{H}_{2} \mathrm{O}\right.$, $2.4 \mathrm{~g} / \mathrm{L} \mathrm{NaCl}$, USA Instruments Inc., $\sigma=0.33 \mathrm{~S} / \mathrm{m}, \varepsilon_{\mathrm{r}}=77.49$, measured at $127.7 \mathrm{MHz}$ with $85070 \mathrm{E}$ dielectric probe, Keysight Technologies). The corresponding arrangement for the array coil is shown in Fig. 5b.
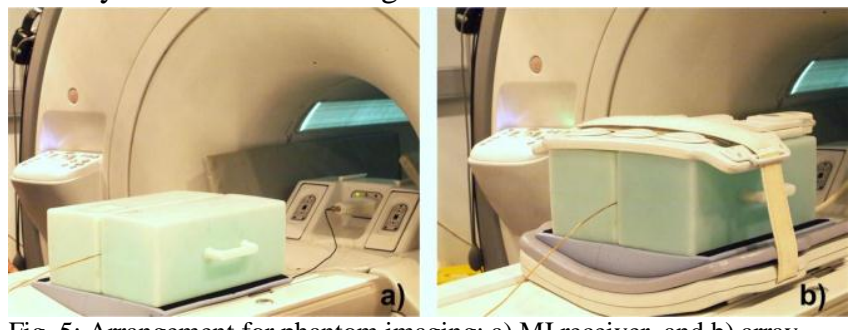

Fig. 5: Arrangement for phantom imaging: a) MI receiver, and b) array.

When imaging with the array, the MI receiver was terminated with a $50 \Omega$ load. The array was removed when the MI receiver was used. Imaging was carried out using a spin echo sequence with the following parameters: repetition time (TR) $400 \mathrm{~ms}$, echo time (TE) $12.9 \mathrm{~ms}$, flip angle $90^{\circ}$, echo train length (ETL) 3, slice thickness $5 \mathrm{~mm}$, slice separation 0.5 $\mathrm{mm}$, field-of-view (FOV) $34 \mathrm{~cm}$, percentage phase FOV 0.6, acquisition matrix 256 (frequency) x 192 (phase), NEX 1, acquisition time $37 \mathrm{~s}$, and pixel bandwidth $\Delta f=122 \mathrm{~Hz}$.

Fig. 6a shows the SNR map of a MI coil axial slice and indicates the horizontal line along which the SNR of the two receivers is compared. The MI receiver has a limited FOV, and its sensitivity falls off rapidly with radius. Two signal regions corresponding to the liquid of the cuboids can be seen. The signal void between extends for $13.2 \mathrm{~mm}$ and includes the catheter diameter and the cuboid wall thickness. Fig. $6 \mathrm{~b}$ shows corresponding results from the array coil and Fig. 6c the SNR variation along the horizontal line for each coil. The array has a large FOV extending across both phantoms, with an average SNR of 120. However, the problem of standing waves [27], which deteriorates the SNR uniformity, is apparent.

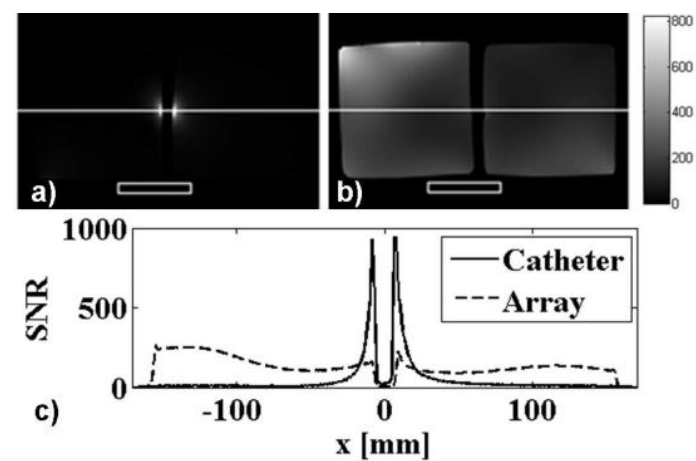

Fig. 6: SNR maps of the axial slices of the phantom: a) magneto-inductive catheter receiver, b) array coil; c) SNR variation along the indicated line.

The pixel SNR has been defined as SNR $=0.66 * \mathrm{~S} / \mathrm{SD}$, where $\mathrm{S}$ is the pixel signal and $\mathrm{SD}$ is the standard deviation of the noise in an $\mathrm{N} \times \mathrm{M}$ (here, $76 \times 8$ ) region-of-interest (ROI) with no signal sources shown at the bottom of the SNR maps. The SD is 3.52 and 6 in the MI and array coil images respectively. The size and location of the ROI do not affect the calculated SNR.

Despite the high loss of the MI cable $(8 \mathrm{~dB} / \mathrm{m})$, the catheter coil outperforms this array coil over a diameter of $32 \mathrm{~mm}$. This is clearly a useful result; it implies that there are circumstances when the MI catheter can win convincingly. Of course comparison with a more optimal array coil design may yield a less favorable result. However, a different array coil would require at least a 5-fold SNR improvement in order to cancel the benefit of the MI catheter receiver completely. To assess the impact of tissue loading, the catheter was also inserted over a length of $35 \mathrm{~cm}$ between the phantoms, resulting in a small but measurable SNR deterioration.

\section{B. Model validation}

Fig. 7a shows the model geometry which exactly mimics the experiment of Fig. 5a. The MI receiver is located between two cuboid phantoms, each of cross-sectional area A. In this arrangement, the entire catheter is naturally outside the region of the conductive body. Fig. 7b shows the corresponding system for the array coil. Its anterior and posterior parts have each been modelled as a 1D array of four coplanar elements. Each element is a pair of long conductors with $\left(2 x_{0}\right)=125 \mathrm{~mm}$ and length $400 \mathrm{~mm}$. The $x$-co-ordinate of each element's centre is $l_{i}$. For both the anterior and posterior coil elements $l_{1,5}=-195 \mathrm{~mm}, l_{2,6}=-65 \mathrm{~mm}, l_{3,7}=65 \mathrm{~mm}$ and $l_{4,8}=195 \mathrm{~mm}$. The $y$-co-ordinate of each element's centre is $k_{i}$ with $k_{1-4}=85$ $\mathrm{mm}$ for the anterior coil elements and $k_{5-8}=-85 \mathrm{~mm}$ for the posterior array coil elements. The space between the external coil conductors and the surface of the cuboid wall is $10 \mathrm{~mm}$. The surface coil geometry we have assumed matches as best as possible the equipment available to us.

We first consider as an example an internal coil with $x_{0}=$ $1.1 \mathrm{~mm}$ and a single external coil with $x_{0}=27.5 \mathrm{~mm}$ (25 times larger). Calculation of $R_{B}$ for each coil is performed using (8). In each case the singularities of the integrand at $y=0$ and $x=$ $\pm x_{0}$ (or at $v=0$ and $u= \pm 1$ in the normalized space) are avoided. The integral converges for small step sizes giving the result that $R_{B}=0.37 \Omega / \mathrm{m}$ and $59.1 \Omega / \mathrm{m}$ for the internal and external coils respectively. The total resistance can be 
estimated by multiplication by the coil length, leading to $R_{B}=$ $0.037 \Omega$ per element for the MI catheter receiver.

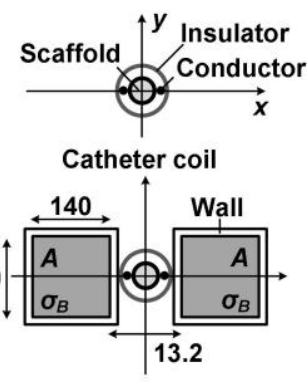

a)

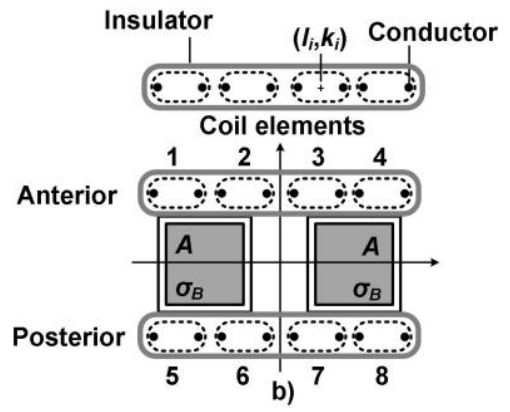

Fig. 7: Model geometries of the experimental arrangements of Fig. 5 for calculation of SNR; a) MI catheter receiver and b) array coil.

The limiting factor in a well-designed detection surface coil is $R_{B}$. However, for a small internal coil, the sample losses are negligible compared to $R_{C}$, typically of the order of $1 \Omega$ for the coils considered here. We also assume that the conductor thickness scales proportionally to the coil radius and therefore $R_{C}$ scales inversely proportionally to the square of the coil radius. Therefore for each element of the surface coil, a conductor resistance of $0.38 \mathrm{~m} \Omega$ is a reasonable estimate.

Following [28] we derive SNR expressions for the 8element surface coil. Based on (9), the RMS value of the NMR signal from a voxel received by each coil element in a $1 \mathrm{D}$ array is $V_{s i}=\left[\omega_{0} v M_{0} / \sqrt{ } 2\right] B_{i}$ where $B_{i}$ is given by:

$$
B_{i}(x, y)=\mu_{0} \sqrt{\left\{H_{x}^{2}\left(x-l_{i}, y-k_{i}\right)+H_{y}^{2}\left(x-l_{i}, y-k_{i}\right)\right\}}
$$

Here $\left(l_{i}, k_{i}\right)$ are the co-ordinates of the centre of each coil. The signal from each element will be phase shifted by an amount $\varphi_{i}$ and amplified by a factor $n_{i}$, leading to a total detected signal $V_{\text {tot }}=S \sqrt{ }\left\{{ }^{\mathrm{N}} \sum_{\mathrm{i}=1}^{\mathrm{N}} \sum_{\mathrm{k}=1}\left[n_{i} n_{k} B_{i} B_{k} \cos \left(\varphi_{i}-\varphi_{k}\right)\right]\right\}$ where $\mathrm{S}$ $=\left[\omega_{0} v M_{0} / \sqrt{2}\right]$.

Since $R_{B}>>R_{C}$ for a surface coil, only body noise is considered. The total resistance can be determined from the time-averaged power dissipated in the sample due to a sinusoidal unit current excitation in the system of $N$ surface coils. Following the derivation in Section II.D, the E-field due to the $\mathrm{i}^{\text {th }}$ coil is: $E_{z i}(x, y)=\left(\mathrm{j} \omega_{0} \mu_{0} I / 4 \pi\right) \log _{\mathrm{e}}\left[\left\{\left(x-l_{i}+x_{0}\right)^{2}+(y\right.\right.$ $\left.\left.\left.k_{i}\right)^{2}\right\} /\left\{\left(x-l_{i}-x_{0}\right)^{2}+\left(y-k_{i}\right)^{2}\right\}\right]$ and the matrix containing the per unit length resistances $R_{i k}$ can be expressed as $R_{i k}=$ $\left(\sigma_{B} \omega_{0}^{2} \mu_{0}^{2} x_{0}^{2} / 16 \pi^{2}\right) C_{i k}$ where $C_{i k}=\iint_{\mathrm{As}} f_{i k} \mathrm{~d} u \mathrm{~d} v$ and $f_{\mathrm{ik}}$ is given by:

$f_{i k}=\log _{e} \frac{\left(u-l_{i n}+1\right)^{2}+\left(v-k_{i n}\right)^{2}}{\left(u-l_{i n}-1\right)^{2}+\left(v-k_{i n}\right)^{2}} \log _{e} \frac{\left(u-l_{k n}+1\right)^{2}+\left(v-k_{k n}\right)^{2}}{\left(u-l_{k n}-1\right)^{2}+\left(v-k_{k n}\right)^{2}}$

Here $l_{i n}=l_{i} / x_{0}, l_{k n}=l_{k} / x_{0}, k_{i n}=k_{i} / x_{0}$ and $k_{k n}=k_{k} / x_{0}$. For $i \neq k$, $R_{i k}$ represents the added noise when coils $i$ and $k$ are used in combination (i.e. the correlated noise). $R_{i i}$ represents the noise due to the $\mathrm{i}^{\text {th }}$ coil alone. The total noise is then given by $R_{t o t}=$ ${ }^{\mathrm{N}} \sum_{i=1}{ }^{N} \sum_{k=1}\left[n_{i} n_{k} R_{i k} \cos \left(\varphi_{i}-\varphi_{k}\right)\right]$ and the SNR of the linear phased array of $N$ elements can be expressed as $\operatorname{SNR}(x, y)=$ $\left|V_{\text {tot }}\right| / \sqrt{ }\left(k_{B} T R_{\text {tot }} \Delta f\right)$. The design objective is to find the optimal combination of gains and phase shifts $a_{i}=n_{i} \mathrm{e}^{\mathrm{j} \rho i}$ that maximize the SNR. Solving the simultaneous equations $\partial \mathrm{SNR}^{2} / \partial n_{i}=0$ and $\partial \mathrm{SNR}^{2} / \partial \varphi_{i}=0$, leads to $\boldsymbol{a}=\lambda \boldsymbol{R}^{-1} \boldsymbol{b}$, with $\boldsymbol{a}, \boldsymbol{b}$ denoting $N \mathrm{x}$ 1 column vectors $a_{i}=n_{i} \mathrm{e}^{\mathrm{j} \varphi i}$ and $b_{i}=B_{i}$ respectively and $\boldsymbol{R}$ an $N$ x $N$ matrix of the noise resistances $R_{i k}$. Here $\lambda$ can be treated as a constant. Substitution of $\boldsymbol{a}$ leads to SNR $=S^{\prime} \sqrt{ }\left\{{ }^{N} \sum_{i=1}^{N} \sum_{k=1}\right.$ $\left.\left[R^{-1}{ }_{i k} b_{i} b_{k}\right]\right\}$ with $S^{\prime}=\left[\omega_{0} v M_{o} / \sqrt{ } 2\right] / \sqrt{ }\left[4 k_{B} T \Delta f\right]$.

We now compare these expressions with the experimental results of III.A. All calculations have been performed in Matlab $^{\circledR}$. The SNR of a catheter coil can be analytically calculated as $\left|V^{\prime}{ }_{s} / V_{n}^{\prime}\right| \times 1 / \sqrt{ } F$. Fig. 8a shows a comparison between the experimental and theoretical SNR for the MI catheter coil along the line in Fig. 6a. It illustrates the numerical result, determined as $\sqrt{ } S_{P} / N_{P}$, where $S_{P}=I_{s N} I_{s N} * Z_{0}$ is the signal power at the output and $N_{P}$ the total noise power assuming uncorrelated noise sources. We have exactly modelled partial immersion, over $10 \mathrm{~cm}$, to match the experiment. Agreement is generally good and verifies a $1 / r^{2}$ reduction in SNR away from the conductors.

The difference between the analytical and numerical SNR models is $2 \%$ when $Z_{O M}=50 \Omega$ and slightly higher for a more realistic value of $Z_{O M}=31 \Omega$. Here, $\mathrm{N}=20, \mathrm{x}_{0}=1.35 \mathrm{~mm}, R_{C}{ }^{\prime}$ $=1 \Omega$ (experimentally derived) $R_{B}{ }^{\prime}=0.062 \Omega$ (calculated). Fig. $8 \mathrm{~b}$ shows the respective comparison for the array coil along the line in Fig. 6b, again indicating good agreement.
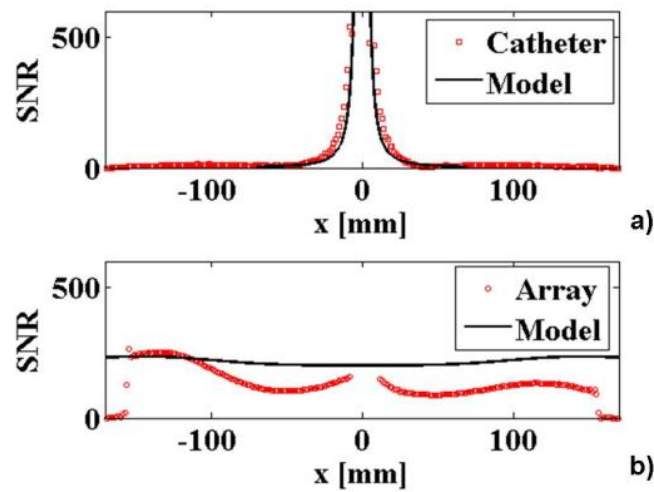

Fig. 8: Theory (model) versus experiment: a) MI catheter receiver (MIC stands for magneto-inductive internal coil) and d) array coil.

\section{Comparison between MI internal receivers and internal coils with conventional cables}

We now perform a theoretical comparison between magneto-inductive receivers and internal coils with conventional cables. We consider the sample geometries shown in Fig. 7 and assume the same electrical, design and imaging parameters as before. The coil with conventional output cable is also a thin-film coil wrapped around a catheter.

We begin by examining light tissue loading. Fig. 9 shows the SNR variation in the horizontal direction for a) the MI coil, with only its tip immersed in tissue, b) an internal coil with conventional cable of the same diameter and c) the array surface coil. Consistent with the experimental results, the catheter coil has a SNR advantage up to a radius of $10 \mathrm{~mm}$. However, as expected, the internal coil with the conventional cable outperforms the MI catheter receiver, and maintains an SNR advantage over the array for radii up to $15 \mathrm{~mm}$. This is however obtained at the price of reduced patient safety. 
Since $R_{B} \ll R_{C}$, theory does not predict a deterioration of SNR due to tissue loading (even when 15 elements are immersed); however, the experimental results do. A likely explanation is the downshift in resonant frequency caused by loading, which will rise with loading. We have verified theoretically that a downshift of $10 \mathrm{MHz}$ in resonant frequency leads to a drop in the SNR of approximately $8 \%$, comparable to the observed deterioration. In practice, detuning will be more complicated due to tissue inhomogeneity. A qualitative ranking of the SNR of the different receiver types which summarizes our findings is shown in Table 1.

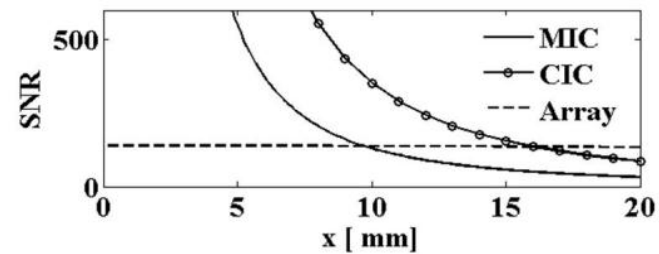

Fig. 9: a) Theoretical longitudinal SNR variation for a MI internal coil (MIC), a conventional internal coil (CIC) and an 8-element array coil.

TABLE I: QUALITATIVE SNR RANKING FOR DIFFERENT COIL SYSTEMS.

\begin{tabular}{cccccc}
\hline \hline Coil System & $R_{B}$ & Intrinsic SNR & $R_{C}$ & $R_{I}$ & Final SNR \\
\hline External & $\mathrm{L}$ & $\mathrm{LO}$ & $\mathrm{S}$ & $\mathrm{S}$ & $\mathrm{LO}$ \\
MIC & $\mathrm{S}$ & $\mathrm{HI}$ & $\mathrm{L}$ & $\mathrm{L}$ & ME \\
CIC & $\mathrm{S}$ & $\mathrm{HI}$ & $\mathrm{L}$ & $\mathrm{S}$ & $\mathrm{HI}$ \\
\hline
\end{tabular}

Key: MIC $=$ Magneto-inductive internal coil, $\mathrm{CIC}=$ Conventional internal coil, $\mathrm{L}=$ Large, $\mathrm{S}=$ Small, $\mathrm{HI}=$ High, $\mathrm{ME}=$ Medium, $\mathrm{LO}=$ Low

\section{DISCUSSION}

We have derived analytical expressions for the SNR of RF receivers based on coils modeled as pairs of long parallel conductors. The validity of the quasi-static approximation is justified since the dimensions of the model considered are smaller than the wavelength at $3 \mathrm{~T}$. We also assumed that the skin depth in the sample is larger than the sample size so that the induced currents do not alter the externally applied RF field [22]. The points of interest do not exceed $75 \mathrm{~mm}$ here hence this approximation is also valid. Our expression for $R_{B}$ is consistent with the result in [17] obtained by ignoring sample skin depth effects. Here the derivation of an analytic expression indicating the dependence on the sample dimensions was not possible. Instead, the dimensions, shape and location with respect to the coils of the sample are all considered numerically in (8). The model was shown to have a good correlation with the experimental data and has confirmed that the experimentally observed small deterioration in the SNR in the heavily loaded case cannot be related to body noise. An internal coil with a standard coaxial cable was found to give the highest SNR, albeit with a risk of RF heating. MI receivers combine a useful SNR advantage with intrinsic safety and therefore provide an excellent compromise, with an SNR advantage over an 8-element surface coil for diameters up to $32 \mathrm{~mm}$ for lengths up to $1 \mathrm{~m}$. Finally Fig. 10a illustrates a more clinically relevant experimental arrangement. Here the catheter is immersed between the cuboid phantoms shown in Fig. 5a over $20 \mathrm{~cm}$ and bent over an angle to emulate cannulation. A corresponding sagittal slice image acquired with the MI catheter receiver is shown in Fig. 10b. The reception pattern is multi-lobed with nulls at the element cross-over points. This provides a first confirmation at $3 \mathrm{~T}$ that bending does not deteriorate the image quality.

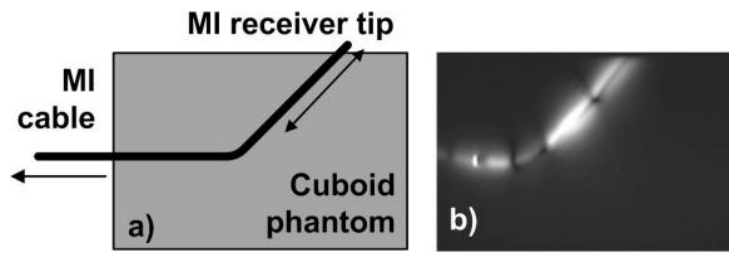

Fig. 10: a) Diagram illustrating a clinically relevant example arrangement of the MI receiver and b) corresponding sagittal image slice.

\section{CONCLUSION}

We have verified the advantage of MI catheter receivers for high SNR, MR-safe imaging at 3T and have derived an intuitive analytical model that can provide further insights in their performance for given design, electrical and sample parameters. The same model was usefully applied to calculate the SNR of different types of receivers, greatly facilitating accurate comparisons without the need to resort to computationally demanding models. Future work will aim at further improving the noise factor of the MI catheter receiver. We will also opt for additional safety tests and SNR comparisons with more optimal array coils. MRI with more clinically relevant samples will be also carried out.

\section{ACKNOWLEDGMENT}

The Authors gratefully acknowledge financial support from the Wellcome Trust.

\section{REFERENCES}

[1] P.A. Bottomley et al.,"Cardiovascular MRI probes for the outside in and for the inside out",Magn. Reson. Mater. Phy., vol.11, pp.49-51, 2000

[2] Y. Eryaman Y, Y. Öner, and E. Atalar, "Design of internal MRI coils using ultimate intrinsic SNR", Magn. Reson. Mater. Phy., vol.22, pp.221-228, 2009

[3] S Sathyanarayana and P.A. Bottomley, "MRI endoscopy using intrinsically localized probes", Med. Phys., vol.36, pp.908-919, 2009.

[4] W.R. Nitz WR et al., "On the heating of linear conductive structures as guide wires and catheters in interventional MRI", J.Magn. Reson. Imaging, vol. 13, pp.105-114, 2001

[5] F.G. Shellock, "Radiofrequency energy-induced heating during MR procedures: a review” J. Magn. Reson. Imag., vol. 12, pp. 30-36, 2000

[6] M.E. Ladd and H. H. Quick, "Reduction of resonant RF heating in intravascular catheters using coaxial chokes", Magn. Reson. Med., vol.43, pp.615-619, 2000

[7] S.Weiss S et al., "Transmission line for improved RF safety of interventional devices",Magn. Reson. Med., vol.54, pp.182-189, 2005.

[8] A. Krafft et al.,"B1 field-insensitive transformers for RF-safe transmission lines",Magn. Reson. Mater. Phy.,vol.19, pp.257-266, 2006

[9] E. Shamonina et al., "Magneto-inductive waveguide", Elect. Lett., vol. 38, 371-373, 2002

[10] R.R.A. Syms et al., "Magneto-inductive catheter receiver for magnetic resonance imaging", IEEE Trans. Biomed. Eng., vol. 60, pp.24212431, 2013

[11] R.R.A. Syms, I.R. Young and M. Rea, "Frequency scaling of catheterbased magneto-inductive MR imaging detectors", Solid-State Sensors, Actuators and Microsystems, Transducers \& Eurosensors XXVII: The 17th International Conference on, pp 594-597, 2013

[12] R. R. A. Syms et al., "Magnetic resonance imaging duodenoscope", IEEE Trans. Biomed. Eng., vol. 60, pp.3458-3467, 2013 
[13] K. Segkhoonthod, R.R.A. Syms and I.R.Young, "Design of magnetoinductive magnetic resonance imaging catheters". IEEE Sensors Journal, vol.14, pp.1505-1513, 2014

[14] R.R.A. Syms et al., "Thin-film magneto-inductive cables". "J. Phys. D: Appl. Phys., vol.43, pp.055102, 2010

[15] R.R.A. Syms and L. Solymar, "Noise in metamaterials", J. Appl. Phys., vol.109, pp.124909, 2011

[16] M. Wiltshire and R.R.A. Syms, "Noise performance of magnetoinductive cables, J. Appl. Phys.,vol.116, pp.034503, 2014

[17] D. Hoult and P.C. Lauterbur, "The sensitivity of the zeugmatographic experiment involving human samples", J. Magn. Reson., vol.34, pp.425-433, 1979

[18] W. Edelstein et al. "The intrinsic signal-to-noise ratio in NMR imaging", Magn. Reson. Med., vol.3, pp.604-618, 1986

[19] M.D. Harpen, "Sample noise with circular surface coils", Med. Phys.,vol.14, pp.616-618, 1987

[20] H. Vesselle and R.E. Collin, "The signal-to-noise ratio of nuclear magnetic resonance surface coils and application to a lossy dielectric cylinder model. I. Theory", IEEE Trans. Biomed. Eng.,vol.42, pp.497506, 1995

[21] O. Ocali and E. Atalar, "Ultimate intrinsic signal-to-noise ratio in MRI”, Magn. Reson. Med., vol.39, pp.462-473, 1998

[22] W. Schnell et al., "Ultimate signal-to-noise-ratio of surface and body antennas for magnetic resonance imaging", Antennas and Propagation, IEEE Transactions on, vol.48, pp.418-428, 2000

[23] H. Çelik et al., "Evaluation of internal MRI coils using ultimate intrinsic SNR", Magn. Reson. Med., vol.52, pp.640-649, 2004

[24] E. Atalar et al. "High resolution intravascular MRI and MRS by using a catheter receiver coil”, Magn. Reson. Med., vol. 36, pp 596-605, 1996

[25] G. Goubau, "Surface waves and their application to transmission lines", J. Appl. Phys.,vol. 21, pp 1119-1128

[26] V. Acikel and E. Atalar,“" Modeling of radiofrequency-induced currents on lead wires during MR imaging using a modified transmission line method", Med. Phys., vol. 28, pp 6623-6632, 2011

[27] T. Skloss, "Phantom fluids for high field MR imaging", Proc. Intl. Soc., Mag. Reson. Med. 11, 1635, 2004

[28] P.Roemer et al. "The NMR phased array", Magn. Reson. Med., vol.16, pp.192-225.1990

Evdokia Kardoulaki received the Ph.D. degree in CMOS filter design from the Department of Bioengineering, Imperial College London, U.K., in 2012. She is currently a postdoctoral researcher in Electrical and Electronic Engineering Department. Her research interests include sensors for biomedical applications, medical imaging and MRI safety.

Richard R.A. Syms is a Professor in the Electrical and Electronic Engineering Department, Imperial College London, London, U.K. His research interests include electromagnetic theory, sensors and medical imaging. He is a Fellow of the Royal Academy of Engineering, the Institute of Electrical Engineers, and the Institute of Physics.

Ian R. Young is a Senior Research Fellow with the Electrical and Electronic Engineering Department, Imperial College London, U.K. His current interests are in vivo MRI and MRguided surgical robots. He is a Fellow of the Royal Society and the Royal Academy of Engineering, and he received the Gold Medal of SMRM and the Sir Frank Whittle Prize.

Marc Rea received the Ph.D. degree in MR-compatible systems for prostate biopsy from Imperial College London, London (NHS Trust, Paddington, London W2 1NY), U.K., in 2010. He is currently a Clinical Scientist at St. Mary's Hospital, London. His research interests include medical robotics, MRI-compatible device tracking, and high-intensity focused ultrasound. 